\title{
Effect of Sequential Isoproturon Pulse Exposure on Scenedesmus vacuolatus
}

\author{
Nathalie Vallotton · Rik Ilda Lambertus Eggen · \\ Nathalie Chèvre
}

Received: 28 February 2008/ Accepted: 5 July 2008/Published online: 15 August 2008

(C) Springer Science+Business Media, LLC 2008

\begin{abstract}
Aquatic organisms are typically exposed to fluctuating concentrations of herbicides in streams. To assess the effects on algae of repeated peak exposure to the herbicide isoproturon, we subjected the alga Scenedesmus vacuolatus to two sequential pulse exposure scenarios. Effects on growth and on the inhibition of the effective quantum yield of photosystem II (PSII) were measured. In the first scenario, algae were exposed to short, 5-h pulses at high isoproturon concentrations (400 and $1000 \mu \mathrm{g} / \mathrm{l}$ ), each followed by a recovery period of $18 \mathrm{~h}$, while the second scenario consisted of 22.5-h pulses at lower concentrations (60 and $120 \mu \mathrm{g} / \mathrm{l}$ ), alternating with short recovery periods $(1.5 \mathrm{~h})$. In addition, any changes in the sensitivity of the algae to isoproturon following sequential pulses were examined by determining the growth rate- $\mathrm{EC}_{50}$ prior to and following exposure. In both exposure scenarios, we found that algal growth and its effective quantum yield were systematically inhibited during the exposures and that these effects were reversible. Sequential pulses to isoproturon could be considered a sequence of independent events. Nevertheless, a consequence of inhibited growth during the repeated exposures is the cumulative decrease in biomass production. Furthermore, in the second scenario, when the sequence of long pulses began to approach a scenario of continuous exposure, a slight increase in the tolerance of the algae to isoproturon was observed. These findings
\end{abstract}

N. Vallotton · R. I. L. Eggen

Eawag, Swiss Federal Institute of Aquatic Science and

Technology, P.O. Box 611, Ueberlandstr. 133, 8600 Duebendorf, Switzerland

N. Chèvre $(\bowtie)$

IPTEH, Faculty of Geociences and Environment, University of Lausanne, 1015 Lausanne, Switzerland

e mail: nathalie.chevre@unil.ch indicated that sequential pulses do affect algae during each pulse exposure, even if algae recover between the exposures. These observations could support an improved risk assessment of fluctuating exposures to reversibly acting herbicides.

Isoproturon is a phenylurea herbicide applied to prevent the development of pre-emergent weeds on cereals fields (wheat and barley). It is one of the herbicides commonly detected in surface waters. The pattern of herbicidal exposure in surface waters varies depending on several factors including the characteristics of the watershed and waterways, the intensity and timing of the rainfall, and the amounts of herbicides used (Berenzen et al. 2005; Garmouma et al. 1998). Concentrations of isoproturon measured in rivers were shown to fluctuate with time. The levels measured by Muller et al. (2002) ranged between 0.05 and $23.18 \mu \mathrm{g} / \mathrm{l}$, with a median at $0.21 \mu \mathrm{g} / \mathrm{l}$. Furthermore, peak concentrations are typically detected following rain events after the isoproturon field application, when this compound is transported from the fields to surface waters by surface runoff, drainage, or spray drift (Kreuger 1998). For example, concentrations in Swiss rivers up to $8.4 \mu \mathrm{g} / \mathrm{l}$ greatly exceeded the suggested Swiss chronic water quality criterion (CQC), set at $0.27 \mu \mathrm{g} / \mathrm{l}$ for this herbicide (Chèvre et al. 2006; Balsiger et al. 2007).

Photosystem II (PSII) inhibiting herbicides, like isoproturon, are known to induce oxidative stress that results in the damage of proteins, lipids, and other cellular components, thus inhibiting growth or leading to plant death (Rutherford and Krieger-Liszkay 2001). Oxidative stress and inhibition of photosynthesis are the consequence of 
blocked electron transfer in PSII, as the secondary plastoquinone acceptor is displaced by the herbicide from the $\mathrm{Qb}$ site of the D1 protein (Rensen 1982). Previous studies have shown that the growth of aquatic plants and algae during pulse exposure to PSII inhibitors was reduced, but their growth recovered after a single exposure (Cedergreen et al. 2005; Drost et al. 2003; Klaine et al. 1997; Vallotton et al. 2008a). In creeks and rivers, the aquatic flora is, however, not exposed solely to a single pulse but, rather, to multiple pulse exposures (Muller et al. 2002; Leu 2003). Nevertheless, little is known about the long-term effects of these sequential peak exposures on algae and water plants.

As reviewed by Reinert et al. (2002), the long-term effects on nontarget organisms of fluctuating pesticide exposure can be a function of the damage sustained during exposure, the capacity of the organisms to recover, and the duration of the recovery period between pulses. Furthermore, sequential exposure to pesticides could lead to an increased or a decreased effect during each subsequent exposure, depending on the organisms tested and the mode of action of the chemical. If a pulse exposure does not influence the response of a subsequent pulse, the sequential exposures may be considered to be completely independent exposures events.

The aim of this study was to investigate the effects of isoproturon sequential pulse exposure on the growth of the algae Scenedesmus vacuolatus, and the effects at the target site of the herbicide, by exposing algae to two different exposure scenarios. In both exposure scenarios, we analyzed the magnitude of effects on algae during each pulse and each recovery period. Growth and inhibition of the PSII effective quantum yield were measured to monitor population level and target site effects of isoproturon, respectively. In addition, dose-response relationships were obtained at the beginning and end of the sequential exposure experiments to assess possible changes in the sensitivity of algae to isoproturon. Finally, the net decrease in biomass production throughout the sequential exposure, compared to the control, was estimated to assess the overall effects of multiple pulses.

\section{Materials and Methods}

\section{Chemicals}

Isoproturon (Isoproturon Pestanal; 99.8\%), 3-(4-isopropylphenyl)-1,1-dimethylurea, was purchased from SigmaAldrich. Stock solutions at $30 \mathrm{mg} / \mathrm{l}$ isoproturon were prepared in the algal culture medium. The concentration of the stock solution was controlled analytically using HPLCMS-MS (Stoob et al. 2005). The concentration of isoproturon in algal growth media was constant during the 72-h algal toxicity test (data not shown). Experimental test concentrations are expressed as nominal concentrations.

\section{Algal Culture Conditions}

The green unicellular alga Scenedesmus vacuolatus (Chlorophyceae; strain 211-8b; Shihira and Krauss, Philadelphia, PA, USA) was purchased from the alga collection of the Institute for Plant Physiology of the University of Göttingen, Germany. The alga was cultured in a sterile inorganic medium prepared as described by Le Faucheur et al. (2005). Batch cultures were grown in $50 \mathrm{ml}$ of medium in sterile 100-ml Erlenmeyer flasks, inoculated with cells grown and maintained on a petri plate. The cultures were maintained on a shaker at $90 \mathrm{rpm}$ and $25^{\circ} \mathrm{C}$ with continuous illumination of $105 \mu \mathrm{E} / \mathrm{m}^{2} / \mathrm{s}$ by cool-white fluorescent lamps.

\section{Experimental Design for Sequential Exposure}

Cultures were started from algal colonies conserved on a petri plate. Cells from this culture that had reached exponential growth were transferred to fresh media to an initial optical density (OD) of 0.05 at $685 \mathrm{~nm}\left(\mathrm{OD}_{685}\right)$, which corresponded to a density of 650,000 cells $/ \mathrm{ml}$. Cultures were exposed to the first isoproturon pulse $24 \mathrm{~h}$ following inoculation. Chemical removal at the end of a pulse was achieved by centrifuging the cultures for $7 \mathrm{~min}$ at $3000 \mathrm{rpm}$ and $25^{\circ} \mathrm{C}$. The supernate was discarded and the algae were resuspended in fresh medium. Centrifugation was repeated a second time, ensuring $99.9 \%$ removal of isoproturon. The removal of isoproturon through centrifugation did not affect algal growth. Following chemical removal, cultures were resuspended to an OD of 0.1 in order to maintain exponential growth throughout the experiment. Controls were treated in the same manner.

The cell density and the PSII effective quantum yield were measured throughout the experiment. The cell density was determined by optical measurement at $685 \mathrm{~nm}$ using a spectrophotometer (Uvikon 930; Kontron Instruments, Munich, Germany) over a range in which OD exhibited a linear correlation with cell density, as determined by microscopic Neubauer chamber cell counts (Brand, Wertheim, Germany). This relationship was shown to be similar for control algal cultures and algae exposed to isoproturon.

The inhibition of PSII effective quantum yield was determined using a dual-channel pulse amplitude modulated fluorometer, called ToxY-PAM (Heinz Walz GmbH, Effeltrich, Germany [Schreiber et al. 2002]). This instrument assesses the effective quantum yield of energy conversion at PSII reaction centers by applying a pulsemodulated light to light-adapted algae (Maxwell and 
Johnson 2000). The chlorophyll fluorescence $F$ and the maximum fluorescence $\mathrm{Fm}$ ' were measured using the instruments default settings. Samples were illuminated with measuring light ( $3 \mathrm{~s}$ ) to determine $F$, while saturation pulses were applied to determine $F m^{\prime}$ ' $470 \mathrm{~nm}$ with a pulse width of $0.4 \mathrm{~s}$ ). Fluorescence measurements were spaced $30 \mathrm{~s}$ apart.

\section{Exposure Scenario 1: Short Pulse with Long Recovery Period, High Isoproturon Concentrations}

Algae were exposed to six 5-h sequential isoproturon pulses with recovery periods of $19 \mathrm{~h}$ (Fig. 1). The exposure concentrations were 400 and $1000 \mu \mathrm{g} / 1$ isoproturon, tested in triplicate with three control cultures. These concentrations were chosen to induce significant effects on growth during a short pulse duration. They are based on the growth response following a 10-h exposure (Vallotton et al. 2008a): $1000 \mu \mathrm{g} / \mathrm{l}$ corresponds to a 10-h EC80 for the growth rate endpoint, while $400 \mu \mathrm{g} / \mathrm{l}$ is greater than a $10-\mathrm{h}$ EC50 for the growth rate endpoint. The growth rate was calculated during each recovery period based on four cell density measurements, but could not be calculated during exposure because of the low number of measurements made during the short pulse. The PSII effective quantum yield was measured prior to each pulse exposure, three times during exposure, and directly following chemical removal.

The change in sensitivity of algae was assessed by comparing the response of the algae to a 24-h exposure to isoproturon at the beginning of the experiment $(t=24 \mathrm{~h})$ and following sequential exposure $(t=150 \mathrm{~h})$. At the beginning of an experiment, a 24-h growth test was started, parallel to the sequential pulse experiment. At the end of the sequential pulse experiment two 24-h tests were conducted with algae pooled from the control cultures and with algae from cultures sequentially exposed to $1000 \mu \mathrm{g} / \mathrm{l}$ isoproturon. In the three 24-h tests, algae were exposed to isoproturon concentrations ranging from 30 to $695 \mu \mathrm{g} / \mathrm{l}$ in a geometric series of 2.85 .

\section{Exposure Scenario 2: Long Pulse with Short Recovery Time, Low Concentrations}

The sequential exposure consisted of five isoproturon pulses, each lasting $22.5 \mathrm{~h}$, with recovery periods of $1.5 \mathrm{~h}$ (Fig. 2). The chosen recovery periods were shorter than the 3-h target-site time to recovery observed following a single-pulse exposure (Vallotton et al. 2008a). Algae were exposed to 60 and $120 \mu \mathrm{g} / \mathrm{l}$ isoproturon: $60 \mu \mathrm{g} / \mathrm{l}$ isoproturon corresponds to a 24-h EC30 for the growth rate endpoint and is less than the 24-h EC50 for the inhibition of effective quantum yield; $120 \mu \mathrm{g} / \mathrm{l}$ isoproturon corresponds to a
(A)
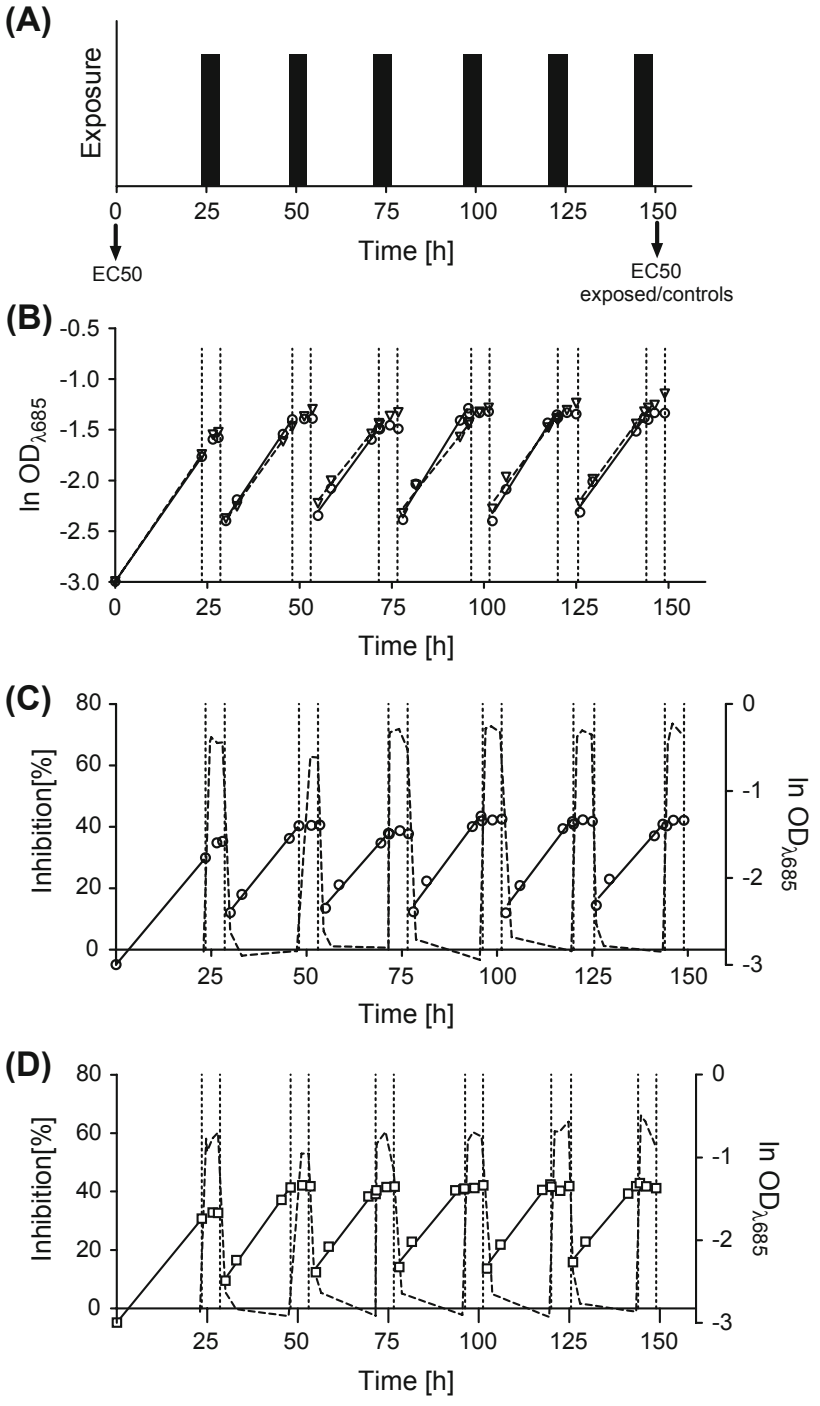

Fig. 1 Short pulses at high isoproturon concentrations. (a) Experi mental design for the sequential exposure to $5 \mathrm{~h}$ pulses (dotted line in b d). The EC50 for algae growth was determined where indicated by the arrows. (b) Effects on cell density and growth of exposures of a Scenedesmus vacuolatus population exposed to $1000 \mu \mathrm{g} / \mathrm{l}$ pulses $(\bigcirc$, solid line) compared to a control $(\nabla$; dashed line). After each chemical removal step, algal cultures were diluted to an optical density of 0.1, which explains the gap in the cell density. (c) Effect of a sequential exposure to $1000 \mu \mathrm{g} / \mathrm{l}$ on cell density and growth between the exposures of a culture $(O$; solid line); inhibition of photosynthetic effective quantum yield of PSII (dashed line). (d) Effect of a $400 \mu \mathrm{g} / \mathrm{l}$ sequential exposure on cell density ( $\square$ ); inhibition of the effective quantum yield of PSII (dashed line)

24-h EC50 for the inhibition of the effective quantum yield. The two exposure levels were tested in triplicate with three control cultures. The effective quantum yield was measured prior to each pulse exposure, four times during exposure, and following chemical removal. The growth rate during the exposure periods was calculated based on five cell density measurements. 

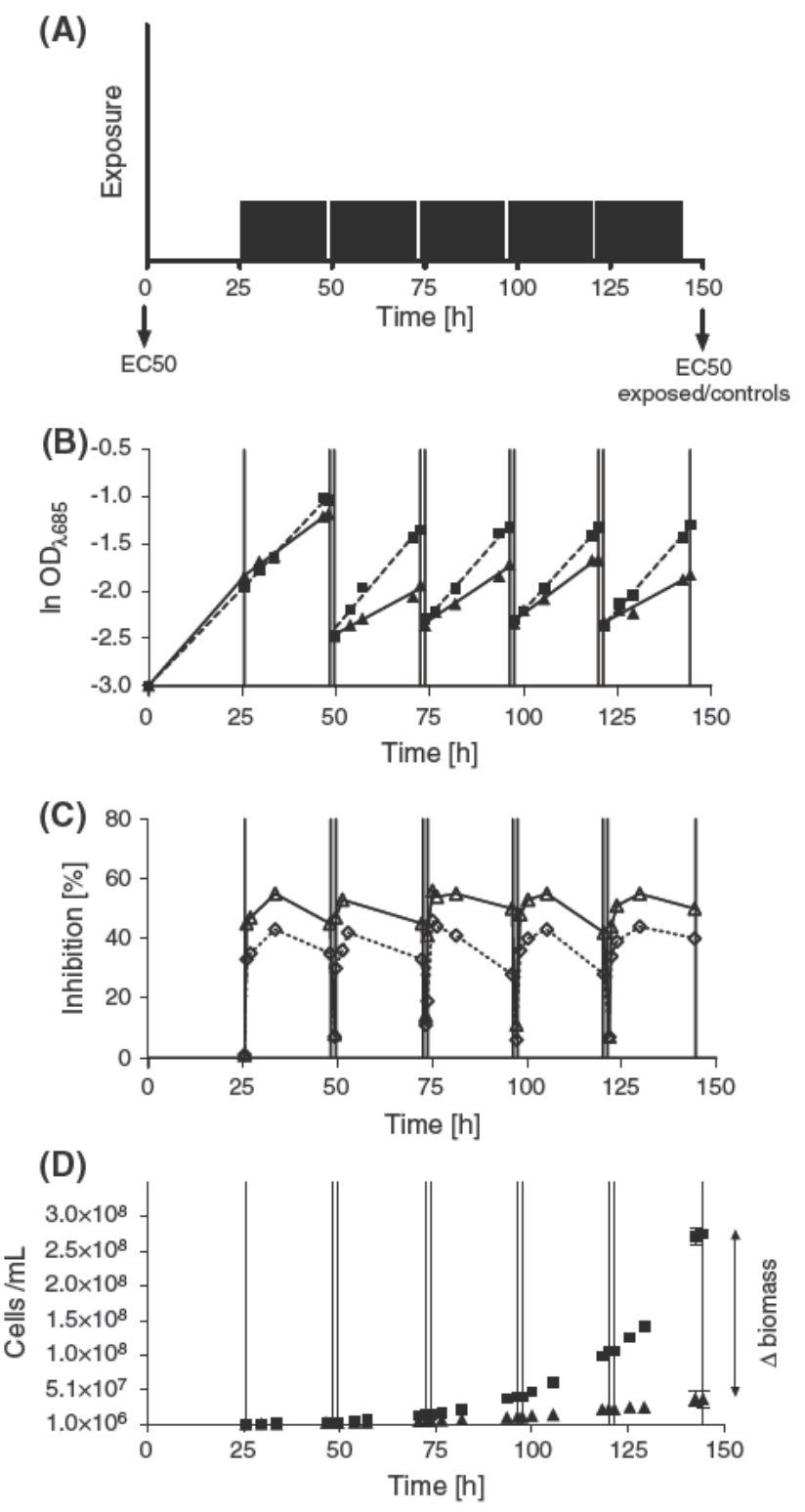

Fig. 2 Long pulses at low isoproturon concentrations with a short recovery time. (a) Experimental design for the sequential exposure to $22.5 \mathrm{~h}$ pulses (bars in a and solid lines in b d). The EC50 for algae growth was determined where indicated by the arrows. (b) Cell density and growth rate regression of a Scenedesmus vacuolatus population exposed to $120 \mu \mathrm{g} / 1$ pulses ( $\boldsymbol{\Delta}$; solid line) compared to a control ( $\square$; dashed line). After each chemical removal step, algal cultures were diluted to an optical density of 0.1 , which explains the gap in the cell density. (c) Inhibition of the PSII effective quantum yield of algae exposed to $60 \mu \mathrm{g} / 1(\diamond$; dotted line) and $120 \mu \mathrm{g} / \mathrm{l}(\Delta$; solid line). (d) Cumulative biomass production during sequential pulses for average controls ( $\square$ ) and cultures sequentially exposed to $120 \mu \mathrm{g} / 1$ isoproturon $(\boldsymbol{\Delta})$. Error bars represent the standard error $(n=$ 3)

As in exposure scenario 1 , the change in the sensitivity of algae was assessed. A 24-h test was performed at the beginning of the experiment. Following sequential exposure to 22.5 -h pulses, a $24-\mathrm{h}$ test could not be run due to the low number of cells available, therefore two 72-h tests were run with algae from the control cultures and with algae from cultures sequentially exposed to $120 \mu \mathrm{g} / 1$ isoproturon. Four concentrations were tested in duplicate with two control cultures.

\section{Data Analysis}

\section{Algal Growth}

The growth rate was calculated as the slope of the linear regression of the natural logarithm of cell density measurements versus time. The inhibition of the growth rate

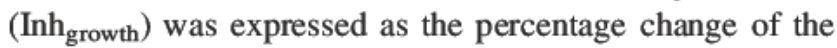
experimental growth rate $\left(\mathrm{GR}_{\mathrm{e}}\right)$ from that of the controls $\left(\mathrm{GR}_{\mathrm{c}}\right)(\mathrm{Eq}$. 1).

$I n h_{\text {growth }}=100 * \frac{G R_{c}-G R_{e}}{G R_{c}}$

The significance of the difference between the average growth rate of the controls and that of the exposed alga was tested with a Student test. The hypotheses were as follows: $\mathrm{H} 0, \mu_{1}=\mu_{2}$; and H1, $\mu_{1} \neq \mu_{2}$.

The biomass production over the entire duration of the experiment (number of cells per milliliter) was calculated to evaluate the cumulative effect of sequential pulse exposure. Since the experimental procedure required the daily dilution of algae, the daily biomass increases between the centrifugation steps were summed. The biomass at $t>$ $t_{x}$ is expressed as

$$
\begin{aligned}
N(t, c)= & N t x * \exp \mu_{c x}\left(t-t_{x}\right) \\
& +\sum_{x>0}^{n} N_{t_{x} 1, c} * \exp \mu_{c x} 1\left(t_{x}-t_{x} 1\right) \\
& -\sum_{x=2,4,6}\left(\left(N_{\left(t_{x}, c\right)} \exp \mu_{c x} 1\left(t_{x}-t_{x} 1\right)\right)-N_{t x}\right)
\end{aligned}
$$

where $x$ corresponds to growth interval. Exposure periods start at $x=(1,3, \ldots)$, while recovery phases start at $x=$ $(0,2,4, \ldots)$.

\section{Dose Response Curves}

The $\mathrm{EC}_{50}$ values for growth rate inhibition and $95 \%$ confidence interval were estimated using a two-parameter logistic model (Eq. 3), with the aid of the statistics software Prism (1992 2003, GraphPad Software, Inc).

$E=\frac{100}{1+10((\log E C 50 \log C) * \text { Hillslope })}$

where $E$ is the endpoint, inhibition of the growth rate; $C$ is the nominal exposure concentration; and Hillslope is the slope of the dose response curve at EC50. 
Difference in the Sensitivity of the Algae Prior to and Following Sequential Exposure

The significance of the difference between the EC50s from two experiments was tested with an $F$-test that compares the goodness of fit of two models by assessing the sum-ofsquares (1992 2003, GraphPad Software, Inc). The hypotheses were as follows: $\mathrm{H}_{0}, \mathrm{EC}_{50} 0_{1}=\mathrm{EC}_{50}$; and $\mathrm{H}_{1}$, $\mathrm{EC} \mathrm{O}_{1} \neq \mathrm{EC}_{20}$. Significant differences in EC50 values would indicate an increased or decreased sensitivity of the algae to isoproturon.

\section{Inhibition of the PSII Effective Quantum Yield}

The fluorescence $F$ and the maximal fluorescence $F m$ ' were each averaged from the three final measurements of five total. The PSII effective quantum yield of a sample, $Y$, is expressed as a percentage of the reduction in average fluorescence, $F$, with respect to the maximal average fluorescence, $F m$,

$Y=\frac{\left(F m^{\prime}-F\right)}{F m^{\prime}}$

and the inhibition, $\operatorname{Inh}(\%)$, is calculated as follows:

$\operatorname{Inh}(\%)=\frac{Y_{1}-Y_{2}}{Y_{1}}$

where $Y_{1}>Y_{2}, Y_{1}$ is the average yield of the controls, and $Y_{2}$ is the yield of the exposed culture.

\section{Results and Discussion}

\section{Exposure Scenario 1}

Figure 1 presents the effects of sequential short pulse exposures at high isoproturon concentrations. During each of the six isoproturon pulses, the growth of the algae exposed to either 400 or $1000 \mu \mathrm{g} / \mathrm{l}$ was inhibited, as indicated by the cessation of the increase in cell density during the exposures (Fig. 1b and c). Inhibition of growth was expected since the chosen exposure concentrations were higher than the growth inhibition EC50 estimated at 142 $\mu \mathrm{g} / \mathrm{l}$ following a $10-\mathrm{h}$ pulse exposure (Vallotton et al. 2008a). Despite qualitative observation, growth inhibition during each exposure could not be quantitatively compared, because the duration of exposure was too short. During the exposures, the effective quantum yield (Fig. 1c and $\mathrm{d}$ ) reached maximum inhibition within $1.5 \mathrm{~h}$ of chemical addition. The effective quantum yield inhibition ranged from $48 \%$ to $66 \%$ at $400 \mu \mathrm{g} / \mathrm{l}$ isoproturon and from 60 to $74 \%$ at $1000 \mu \mathrm{g} / \mathrm{l}$. These effect levels were similar to the effects observed during single-pulse exposure experiments (Vallotton et al. 2008a). Inhibition of the effective quantum yield during each pulse was relatively constant, which indicated that previous pulse exposures did not influence the response during subsequent pulses. The inhibition of algal growth during pulses can be attributed to the rapid onset of inhibitory effects at the target site.

Similar to the fast onset of effects following chemical addition, fast recovery of the effective quantum yield was repeatedly observed within $3 \mathrm{~h}$ after chemical removal. This is in opposition to the reduced and delayed recovery observed for the seagrass Zostra capricorni during the 3day period following two sequential exposures to the triazine herbicide Irgarol (Macinnis-Ng and Ralph 2004). Plants individuals might be more sensitive to sequential exposures than algal populations, which are composed of evolving algal individuals due to cell division. In parallel to the systematic recovery of effects at the target site, we observed a recovery of algal growth between the pulses similar to that observed after single-pulse exposures to PSII inhibitors in algae and duckweed (Cedergreen et al. 2005; Drost et al. 2003; Klaine et al. 1997; Vallotton et al. 2008a). In the experiments here, the average growth rate of the controls was not significantly different from that of the exposed cultures $(p>0.05$; Table 1$)$, except in recovery period $4(p=0.022)$, where the average growth rate of the exposed population was unexpectedly higher. However, neither an increasing nor a decreasing trend in average growth rates was observed during subsequent exposures.

Table 1 Exposure scenario 1: average (and standard error) growth rate during recovery periods of three replicate controls and three experi mental cultures sequentially exposed to $5 \mathrm{~h}$ pulses

\begin{tabular}{|c|c|c|c|c|c|}
\hline & Recovery 1 & Recovery 2 & Recovery 3 & Recovery 4 & Recovery 5 \\
\hline Controls & $0.051(0.001)$ & $0.049(0.002)$ & $0.048(0.003)$ & $0.049(0.002)$ & $0.049(0.001)$ \\
\hline $400 \mu \mathrm{g} / \mathrm{l} \mathrm{IPU}$ & $0.056(0.003)$ & $0.056(0.003)$ & $0.050(0.002)$ & $0.057(0.001)$ & $0.050(0.002)$ \\
\hline$t$ test with control, $p$ value ${ }^{\mathrm{a}}$ & 0.17 & 0.15 & 0.56 & 0.02 & 0.97 \\
\hline $1000 \mu \mathrm{g} / \mathrm{l} \mathrm{IPU}$ & $0.056(0.004)$ & $0.054(0.003)$ & $0.056(0.002)$ & $0.058(0.002)$ & $0.053(0.002)$ \\
\hline$t$ test with control, $p$ value ${ }^{\mathrm{a}}$ & 0.30 & 0.24 & 0.12 & 0.02 & 0.17 \\
\hline
\end{tabular}

${ }^{a}$ The significance of the difference in the average growth rates of the two groups ( $p$ value) was tested with a $t$ test versus the controls 

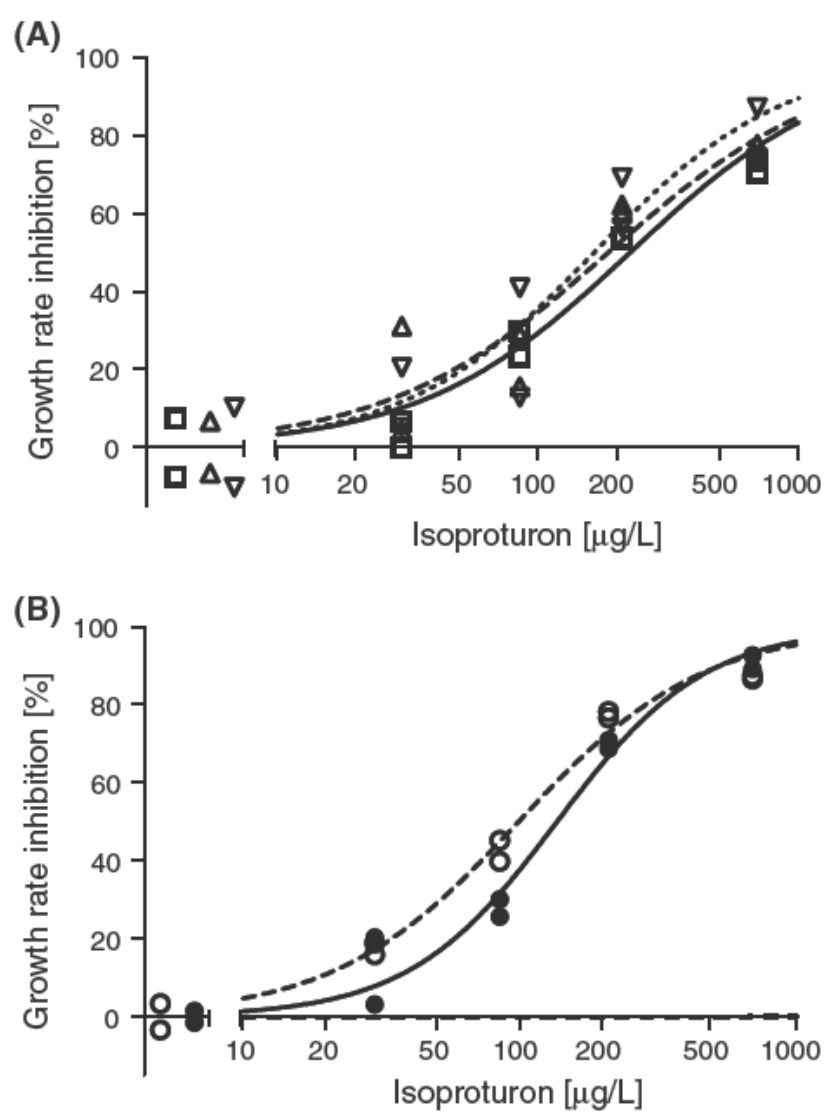

Fig. 3 Dose response curves of $24 \mathrm{~h}$ tests carried out with $S$. vacuolatus before ( $\square$; solid line) and after the six $5 \mathrm{~h}$ sequential pulse experiment (a): nonexposed algae ( $\Delta$; dashed line) and algae sequentially exposed at $1000 \mu \mathrm{g} / 1$ ( $\nabla$; dotted line). (b) Dose response curves of $72 \mathrm{~h}$ tests carried out at the end of exposure scenario 2, with cultures repeatedly exposed to $22.5 \mathrm{~h}$ pulses at $120 \mu \mathrm{g} / \mathrm{l}$ ( $)$; solid line) and with algae used as controls ( $\mathrm{O}$; dashed line)

The evolution in sensitivity of control and sequentially exposed algae was tested by determining the EC50 from 24-h toxicity tests (Fig. 3a). The EC50 of the test performed at the beginning of the experiment was $225 \mu \mathrm{g} / \mathrm{l}$, with a $95 \% \mathrm{CI}$ of $(169,300)$, and the Hillslope 1.08 (0.7334 1.431). The growth rates of controls were 0.047 and $0.040 \mathrm{~h}^{-1}$. At the end of sequential exposure, the EC50 obtained from a 24-h test performed with control algae was estimated at $177 \mu \mathrm{g} / 1(105,296)$, while the EC50 of a second test run with algae that had undergone sequential $1000 \mu \mathrm{g} / \mathrm{l}$ isoproturon pulses was $164 \mu \mathrm{g} / \mathrm{l}(103$, 261). Hillslopes were $1.02(0.437,1.60)$ and $1.19(0.5126$, 1.872 ), respectively. The growth rates of controls were 0.035 and 0.043 in the first run and 0.042 and 0.048 in the second run. The EC50 values of these three tests were not significantly different $(p=0.43)$, indicating that the population that underwent sequential exposure was neither more sensitive nor more resistant to isoproturon.

\section{Exposure Scenario 2}

Figure 2 presents the effects of sequential long pulse exposures with short, 1.5 -h recovery periods on the growth of $S$. vacuolatus and the inhibition of effective quantum yield (Fig. 2a). During each pulse exposure, isoproturon inhibited the growth rate of the exposed algal cultures. For example (Fig. 2b), the growth rate during the pulse exposures of a culture exposed to $120 \mu \mathrm{g} / \mathrm{l}$ ranged from 0.021 to $0.029 \mathrm{~h}^{-1}$, while that of the control varied between 0.042 and $0.047 \mathrm{~h}^{-1}$. Generally (Table 2), the average growth rates of the three controls ranged from 0.041 to $0.047 \mathrm{~h}^{-1}$ during the pulse exposures, while that of cultures exposed to 60 and $120 \mu \mathrm{g} / 1$ were reduced to 0.0360 .043 and to $0.0210 .029 \mathrm{~h}^{-1}$, respectively. Consequently, growth rates were inhibited by $220 \%$ at $60 \mu \mathrm{g} / 1$ and by $2954 \%$ at 120 $\mu \mathrm{g} / \mathrm{l}$. The average inhibition of the growth rate during the first pulse was not significantly different from the average inhibition during the last pulse $(60 \mu \mathrm{g} / 1, p=0.63 ; 120 \mu \mathrm{g} / \mathrm{l}$, $p=0.22$ ). Yet the levels of inhibition during sequential pulses were not influenced by previous exposures despite subsequent exposure prior to full recovery to the pulse exposure (Vallotton et al. 2008a).

As in exposure scenario 1, the effective quantum yield of the exposed culture was inhibited directly following chemical addition and the response was similar among subsequent pulses (Fig. 2c). Within $0.5 \mathrm{~h}$, the effective quantum yield was inhibited by $30 \%$ at $60 \mu \mathrm{g} / 1$ and by more than $40 \%$ at $120 \mu \mathrm{g} / \mathrm{l}$. Inhibition of the effective quantum yield further increased during the first $7 \mathrm{~h}$ of the

Table 2 Exposure scenario 2: average growth rate, with standard error (SE), during exposure of three replicate control and three experimental cultures, sequentially exposed to $22.5 \mathrm{~h}$ pulses at 60 and $120 \mu \mathrm{g} / 1$

\begin{tabular}{llllll}
\hline & Pulse 1 & Pulse 2 & Pulse 3 & Pulse 4 & Pulse 5 \\
\hline Controls & $0.043(0.002)$ & $0.047(0.001)$ & $0.044(0.002)$ & $0.041(0.001)$ & $0.043(0.001)$ \\
$60 \mu \mathrm{g} / \mathrm{IPU}$ & $0.036(0.001)$ & $0.037(0.001)$ & $0.043(0.001)$ & $0.037(0.002)$ & $0.037(0.003)$ \\
Inhibition (\%) & $17(2)$ & $20(1)$ & $2(2)$ & $10(5)$ & $14(6)$ \\
$120 \mu \mathrm{g} / \mathrm{I} \mathrm{IPU}$ & $0.026(0.002)$ & $0.021(0.001)$ & $0.028(0.001)$ & $0.029(0.001)$ & $0.022(0.002)$ \\
Inhibition (\%) & $39(4)$ & $54(1)$ & $37(1)$ & $29(1)$ & $49(5)$ \\
\hline
\end{tabular}

Note: The inhibition of the growth rate is calculated for each pulse exposure 
pulse exposures, reaching a maximum inhibition of 42 $46 \%$ at $60 \mu \mathrm{g} / \mathrm{l}$ and $5158 \%$ at $120 \mu \mathrm{g} / \mathrm{l}$. Furthermore, the response at the target site was highly consistent between exposure concentrations and expected concentrationresponse. It then decreased slightly over the next $12 \mathrm{~h}$ of exposure. We hypothesize that the decrease in effects at the target site during the exposures might be attributed to a decrease in the dose of isoproturon per alga, since the cell density increased during the 22.5-h pulse. During the recovery period, the inhibition of the effective quantum yield dropped below 20\%. The fast recovery between exposures may be attributed to the reversible binding at the target site and to repair mechanisms in PSII that replace the damaged D1 protein in PSII. In Chlamydomonas reinhardtii, following exposure to high-intensity illumination, synthesis of D1 and its incorporation into functional PSIIs were estimated to take $1 \mathrm{~h}$ (Baker and Bowyer 1994; Devine et al. 1993). This repair interval is of the same order of magnitude as the recovery period observed for effective quantum yield inhibition.

Despite the very short recovery periods, the levels of inhibition observed during sequential pulses were not influenced by previous exposures. Furthermore, the pattern of inhibition was comparable among pulses, supporting the hypothesis that longer pulse events $(22.5 \mathrm{~h})$ with short recovery periods could also be considered to be independent, as opposed to cumulative, exposure events. However, the EC50 estimated at the end of exposure for algae that had been sequentially exposed (EC50 $\left.{ }_{\mathrm{SE}}\right)$ was significantly higher than the 72-h EC50 obtained for control cultures (EC50 ${ }_{\mathrm{CC}}, p=0.01$; Fig. 3b). EC50 ${ }_{\mathrm{SE}}$ values were $136 \mu \mathrm{g} / \mathrm{l}$ $(110,169)$ with a Hillslope of $1.311(1.918,2.076)$ and control growth rates of 0.0431 and 0.0418 . EC50 ${ }_{\mathrm{CC}}$ was 99 $\mu \mathrm{g} / \mathrm{l}(83,119)$ with a Hillslope of $1.624(2.042,2.229)$ and control growth rates of 0.0458 and 0.0428 . The difference in EC50 values indicates a slight shift in sensitivity of the response of $S$. vacuolatus to isoproturon toxicity. The increase in EC50 suggested that sequential pulse exposures with short recovery periods may induce a physiological adaptation of the algal population, either by inducing a selection pressure on the population or by enhancing defense mechanisms against oxidative stress. This is in line with the increased tolerance observed in several Scenedesmus subspicatus clones, following a 60-day exposure to atrazine (1 $20 \mu \mathrm{g} / \mathrm{l}$ (Behra et al. 1999). More generally, algal community tolerance increased following long-term exposure to $540 \mu \mathrm{g} / \mathrm{l}$ isoproturon (Peres et al. 1996; Schmitt-Jansen and Altenburger 2005a; Schmitt-Jansen and Altenburger 2005b).

In both scenarios, the growth of algae was inhibited during each exposure, resulting in cumulative losses in biomass production. In exposure scenario 2 (Fig. 2d) the biomass was seven times greater in the controls compared to that of sequentially exposed cultures at $145 \mathrm{~h}$. This loss in biomass production might influence the competitiveness of species in surface water communities, e.g., periphyton (Blanck 2002). Single-pulse exposure (24 and $48 \mathrm{~h}$ ) to the triazine metribuzin was shown to induce a change in species composition and a loss of biomass by different algal groups (Gustavson et al. 2003).

\section{Conclusion and Outlook}

Based on the pulse and recovery intervals tested here, sequential pulse exposure to isoproturon could be considered a sequence of independent events that impaired algal growth during exposure. The effects at the target site and on the growth of $S$. vacuolatus were highly reversible upon chemical removal, even after multiple pulses. Similar effects and recovery might be expected for other herbicides that inhibit PSII. First, the overall consequence of sequential exposures to this highly reversibly acting herbicide was a decreased net biomass production, which would be predictable based on the effects determined from single-pulse exposure experiments. Second, a slight increase in the tolerance of $S$. vacuolatus was observed when the pulse exposures were of the order of 1 day and recovery durations were short. Both effects may alter the competitiveness of some algae species in surface water communities. Additionally, greater effects during sequential exposures to less reversibility acting herbicides, such as chloroacetanilides (Vallotton et al. 2008b), could be expected, since the effect of a first pulse might influence the response to a second pulse. The observation made during each exposure and the resulting cumulative effects over time should support an improved risk assessment of herbicides during fluctuating exposure.

Acknowledgments This work was funded by Swiss National Research Foundation Grant 200021 109569/1. The authors thank Alfred Lück of the Department of Environmental Chemistry, Zachariah Schreiber, and the anonymous reviewers.

\section{References}

Baker NR, Bowyer JR (1994) Photoinhibition of photosynthesis: from molecular mechanisms to the field. Bios Scientific, Oxford

Balsiger C, Niederhauser P, Jäggi O, Meier W (2007) Gewässerbe lastung durch Pestizide. Gaz Wasser Abwasser (GWA) 3:177 185

Behra R, Genoni GP, Joseph AL (1999) Effect of atrazine on growth, photosynthesis, and between strain variability in Scenedesmus subspicatus (Chlorophyceae). Arch Environ Contam Toxicol 37:36 41. doi:10.1007/s002449900487

Berenzen N, Lentzen Godding A, Probst M, Schulz H, Schulz R, Liess M (2005) A comparison of predicted and measured levels 
of runoff related pesticide concentrations in small lowland streams on a landscape level. Chemosphere 58:683 691. doi: 10.1016/j.chemosphere.2004.05.009

Blanck H (2002) A critical review of procedures and approaches used for assessing pollution induced community tolerance (PICT) in biotic communities. Hum Ecol Risk Assess 8:1003 1034. doi: 10.1080/1080 700291905792

Cedergreen N, Andersen L, Olesen CF, Spliid HH, Streibig JC (2005) Does the effect of herbicide pulse exposure on aquatic plants depend on K ow or mode of action? Aquat Toxicol 71:261 271. doi:10.1016/j.aquatox.2004.11.010

Chèvre N, Loepfe C, Singer H, Stamm C, Fenner K, Escher B (2006) Including mixtures in the determination of water quality criteria for herbicides in surface water. Env Sci Technol 40:426 435

Devine MD, Duke SO, Fedtke C (1993) Physiology of herbicide action. Prentice Hall, Englewood Cliffs, NJ

Drost W, Backhaus T, Vassilakaki M, Grimme LH (2003) Mixture toxicity of s triazines to Lemna minor under conditions of simultaneous and sequential exposure. Fresenius Environ Bull 12:601 607

Garmouma M, Teil MJ, Blanchard M, Chevreuil M (1998) Spatial and temporal variations of herbicide (triazines and phenylureas) concentrations in the catchment basin of the Marne river (France). Sci Total Environ 224:93 107. doi: 10.1016/S0048 9697(98)00326 X

Gustavson K, Mohlenberg F, Schluter L (2003) Effects of exposure duration of herbicides on natural stream periphyton communities and recovery. Arch Environ Contam Toxicol 45:48 58. doi: 10.1007/s002440020079 9

Klaine SJ, Richards P, Baker D, Naddy R, Brown T, Joab B, Casey R, Fernandez D, Overmeyer J, Benjamin R (1997) Agrochemical fate and effects in terrestrial, aquatic and estuarine ecosystems. Environmental Behavior of Crop Protection Chemicals, Interna tional Atomic Energy Agency, Vienna, Austria, pp 247263

Kreuger J (1998) Pesticides in stream water within an agricultural catchment in southern Sweden, 1990 1996. Sci Total Environ 216:227 251. doi:10.1016/S0048 9697(98)00155 7

Le Faucheur S, Behra R, Sigg L (2005) Phytochelatin induction, cadmium accumulation, and algal sensitivity to free cadmium ion in Scenedesmus vacuolatus. Environ Toxicol Chem 24:1731 1737. doi:10.1897/04 394R.1

Leu C (2003) Sources, processes and factors determining the losses of atrazine, dimethenamid and metolachlor to surface waters: a simultaneous assessment in six agriculture catchments. Ei dgenössische Technische Hochschule Zürich (ETHZ), Zurich, Switzerland

Macinnis Ng CMO, Ralph PJ (2004) In situ impact of multiple pulses of metal and herbicide on the seagrass, Zostera capricorni. Aquat Toxicol 67:227 237. doi:10.1016/j.aquatox.2004.01.012

Maxwell K, Johnson GN (2000) Chlorophyll fluorescence a practical guide. J Exp Bot 51:659 668. doi:10.1093/jexbot/51.345.659
Muller K, Bach M, Hartmann H, Spiteller M, Frede H G (2002) Point and nonpoint source pesticide contamination in the Zwester ohm catchment, Germany. J Environ Qual 31:309 318

Peres F, Florin D, Grollier T, FeurtetMazel A, Coste M, Ribeyre F, Ricard M, Boudou A (1996) Effects of the phenylurea herbicide isoproturon on periphytic diatom communities in freshwater indoor microcosms. Environ Pollut 94:141 152. doi: 10.1016/S0269 7491(96)00080 2

Rensen JJS (1982) Molecular mechanisms of herbicide action near photosystem II. Plant Physiol 54:515 521

Reinert KH, Giddings JA, Judd L (2002) Effects analysis of time varying or repeated exposures in aquatic ecological risk assess ment of agrochemicals. Environ Toxicol Chem 21:1977 1992. doi :10.1897/1551 5028(2002)021<1977:EAOTVO > 2.0.CO;2

Rutherford AW, Krieger Liszkay A (2001) Herbicide induced oxi dative stress in photosystem II. Trends Biochem Sci 26:648 653. doi:10.1016/S0968 0004(01)01953 3

Schmitt Jansen M, Altenburger R (2005a) Toxic effects of isoprotu ron on periphyton communities a microcosm study. Estuar Coast Shelf Sci 62:539 545. doi:10.1016/j.ecss.2004.09.016

Schmitt Jansen M, Altenburger R (2005b) Predicting and observing responses of algal communities to photosystem II herbicide exposure using pollution induced community tolerance and species sensitivity distributions. Environ Toxicol Chem 24:304 312. doi:10.1897/03 647.1

Schreiber U, Muller JF, Haugg A, Gademann R (2002) New type of dual channel PAM chlorophyll fluorometer for highly sensitive water toxicity biotests. Photosynth Res 74:317 330. doi: 10.1023/A:1021276003145

Stoob K, Singer HP, Goetz CW, Ruff M, Mueller SR (2005) Fully automated online solid phase extraction coupled directly to liquid chromatography tandem mass spectrometry quantifica tion of sulfonamide antibiotics, neutral and acidic pesticides at low concentrations in surface waters. J Chromatogr A 1097:138 147. doi: $10.1016 /$ j.chroma.2005.08.030

Vallotton N, Eggen RIL, Escher BI, Krayenbuhl J, Chevre N (2008a) Effect of pulse herbicidal exposure on Scenedesmus vacuolatus: a comparison of two photosystem II inhibitors. Environ Toxicol Chem 27:1399 1407. doi:10.1897/07 197.1

Vallotton N, Moser D, Eggen RIL, Junghans M, Chèvre N (2008b) S metolachlor pulse exposure on the alga Scenedesmus vacuolatus: effect during exposure and the subsequent recovery. Chemo sphere (in press) 